\title{
Aggregation of Operators of Fuzzy Relational Mathematical Morphology: Erosion and Dilation
}

\author{
Alexander Šostak ${ }^{a, b}$ and Ingrīda Uljane ${ }^{a, b}$ \\ ${ }^{a}$ Institute of Mathematics and CS University of Latvia, Raina bulvāris 29, Riga, Latvia \\ aleksandrs.sostaks@lumii.lv, ingrida.uljane@lumii.lv \\ ${ }^{b}$ Department of Mathematics, University of Latvia, Jelgavas iela 3, Riga, Latvia \\ aleksandrs.sostaks@lu. lv, ingrida.uljane@lu. lv
}

\begin{abstract}
Revising the definitions of fuzzy relational erosion and dilation introduced by $\mathrm{N}$. Madrid et al. ( $L$-fuzzy relational mathematical morphology based on adjoint triples. Inf. Sci. 474, 75-89, 2019), we define the structured versions of these operators and study their basic properties. Our principal interest is aggregation of fuzzy relational, specifically structured, erosions and dilations. We base such aggregation on a dual pair of binary operators $(\diamond, \odot)($ e.g. a $t$-norm and the $t$-conorm); $\diamond$ is applied for aggregation of erosions and $\odot$ for aggregation of dilations.
\end{abstract}

Keywords: structured fuzzy relational erosion, structured fuzzy relational dilation, aggregation, duality, adjunction.

\section{Introduction}

Mathematical morphology has its origins in geological problems centered in the processes of erosion and dilation. The founders of mathematical morphology are engineers G. Matheron [10] and J. Serra [13]. The idea of the classical mathematical morphology can be explained as the process of modifying a subset $A$ of a cube in an n-dimensional Euclidean space $\mathbb{R}^{n}$ by cutting out pieces of $B$ from $A$ (in case of erosion) or glueing them down to the set $A$ (in case of dilation). The set $B$, intuitively, small if compared with $A$, is called the structuring element. In the first works on fuzzy morphology, $A$ and $B$ were crisp sets, however soon the interest of some researchers was directed also to the case when $A$ and $B$ could be fuzzy. This allowed to describe grey scale processes of erosion and dilation. The first fundamental works on fuzzy mathematical morphology are papers by B. De Baets, E. Kerre and M. Gupta [3]. Later much work has been done in fuzzy mathematical morphology in the framework of an Euclidean space, a detailed survey of different approached to the subject of mathematical morphology, in particular fuzzy, is presented in [7].

In the middle nineties of the previous century some researches were attracted by the prospects to have a general abstract approach to (fuzzy) mathematical morphology, that would not depend on the specific linear structure of the Euclidean space. As the background for this theory complete distributive lattices sometimes with an additional algebraic structure were usually taken. Concerning the properties of erosion and dilation that should be preserved in any generalized theory, the two fundamental features of the classical mathematical morphology were taken as a basis. Namely, they are the duality between erosion and dilation and the adjunction of this pair of operators. The detailed survey and analysis of this general approach to fuzzy mathematical morphology and the related questions is contained in the paper [1].

In a recent paper, [9] the authors applied this general algebraic approach in case when erosion and dilation operators are defined by means of an $L$-fuzzy relation which is defined on a set $X$ and takes values in $L$. This approach in some sense can be viewed as intermediate between the abstract algebraic approach and the "classical" one: the $L$-fuzzy relation $R$ laid in the base of this approach determines a structure on a set $X$, and that can be viewed as a certain substitute of the linear structure in Euclidean space. This approach was further developed in [14].

While being very well interpreted as an important specification of the general algebraic approach to fuzzy mathematical morphology, this approach still misses an essential feature of the "classical" one. Namely, while fuzzy relation $R$ may be considered as a substitute for the linear structure of the Euclidean space, the fuzzy relational approach misses the second important ingredient of the classic approach, namely the 
structuring element $B$. Of course, one may assume that the fuzzy relation $R$ is a combined substitute both of the linear structure of $\mathbb{R}^{n}$ and the structuring element. However, in our opinion, this is not a natural interpretation, neither it is appropriate for different manipulations with fuzzy morphological operators, in particular in the process of aggregation. In order to overcome this problem, in [15] we proposed a structured version of fuzzy relational morphological operators. It assumes intermediate use of the product and co-product in the definition of erosion and dilation and this created inconvenience in the study and especially in the use of such operators. As different from that approach, here we define structured versions of relational erosion and dilation operators by incorporating the structuring element $B$ in the local modification of the original $L$-fuzzy relation; this seems to be more natural and convenient for the manipulation with operators.

The paper consists of five sections, including this introduction and conclusion. In the second, preliminary section, basic concepts and results used in this paper are expounded. In the third section, we define fuzzy relational structured erosion and dilation and investigate their basic properties and interrelations. In the fourth section, consisting of four subsections we define and study some schemes for aggregation fuzzy erosion and dilation. These schemes are based on a pair $(\diamond, \odot)$ where a product (specifically a $t$-norm) $\diamond$ is used for aggregation of erosion operators while the co-product $\odot$ is used for aggregating of a dilation operators. In conclusion section we sketch some directions were aggregation theory initiated here should be continued.

\section{Preliminaries}

In this section, we recall some concepts from the theory of lattices (see e.g. [4]), quantales (see e.g. [12]) and $L$-fuzzy relations (see e.g. [17], [16]) and adapt them in a way appropriate for the context of our work.

2.1. Lattices, quantales. In our paper, $(L, \leq, \wedge, \vee)$ is a complete infinitely distributive lattice with bottom and top elements $0_{L}$ and $1_{L}$ respectively. Given a binary commutative associative monotone operation $*: L \times L \rightarrow L$, the tuple $(L, \leq, \wedge, \vee, *)$ is called $a$ (commutative) quantale if $*$ distributes over arbitrary joins: $a *\left(\bigvee_{i \in I} b_{i}\right)=\bigvee_{i \in I}\left(a * b_{i}\right)$. Operation $*$ will be referred to as the product in $L$. Product $*$ is called meetdistributive if it distributes over arbitrary meets, that is $a *\left(\bigwedge_{i \in I} b_{i}\right)=\bigwedge_{i \in I}\left(a * b_{i}\right)$. A quantale is called integral if the top element $1_{L}$ acts as the unit, that is $1_{L} * a$ for every $a \in L$. In what follows saying quantale we mean a commutative integral quantale.

In a quantale a further binary operation $\mapsto: L \times L \rightarrow L$, the residuum, can be introduced as associated with operation $*$ of the quantale $(L, \leq, \wedge, \vee, *)$ via the Galois connection, that is $a * b \leq c \Longleftrightarrow a \leq b \mapsto c$ for all $a, b, c \in L$. We need the following known property of quantales:

Lemma 2.1 (see, e.g. [5].) In a quantale $a \mapsto(b \mapsto$ $c)=a * b \mapsto c$ for any $a, b, c \in L$.

Proof $a \mapsto(b \mapsto c)=\bigvee\{\lambda \mid \lambda * a \leq b \mapsto c\}=\bigvee\{\lambda \mid$ $\lambda * a * b \leq c\}=a * b \mapsto c$.

2.2. De Morgan quantales One of the two fundamental properties of (fuzzy) mathematical morphology is duality between its two basic operators: erosion and dilation. In order to consider the counterpart of this property in the framework of our approach, we have to enrich the background of research with an order reversing involution. Recall that an unary operator ${ }^{c}: L \rightarrow L$ is called negation if it is an order reversing involution, that is $a \leq b \Longrightarrow b^{c} \leq a^{c}$ and $\left(a^{c}\right)^{c}=a$ for all $a, b \in L$. A lattice $L$ endowed with a negation that is the tuple $\left(L, \leq, \wedge, \vee,^{c}\right)$ is called a De Morgan algebra; respectively, the tuple $\left(L, \leq, \wedge, \vee, *{ }^{c}\right)$ will be referred to as a De Morgan quantale. In a De Morgan quantale a further binary operation, co-product $\oplus$, can be defined by setting $a \oplus b=\left(a^{c} * b^{c}\right)^{c}$ for all $a, b \in L$. Co-product is a commutative associative monotone operation and, in case $(L, \leq, \wedge, \vee, *)$ is integral, $0_{L}$ acts as a zero, that is $a \oplus 0_{L}=a$ for every $a \in L$. Important properties of De Morgan quantales are collected in the next lemmas:

Lemma 2.2 [15] Operation $\oplus$ in a De Morgan quantale $(L, \leq, \wedge, \vee, *)$ is meet-distributive: $a \oplus\left(\bigwedge_{i \in I} b_{i}\right)=\bigwedge_{i \in I}\left(a \oplus b_{i}\right) \quad \forall a \in L, \forall\left\{b_{i} \mid i \in I\right\} \subseteq L$.

Lemma 2.3 [15] If operation * in a De Morgan quantale $(L, \leq, \wedge, \vee, *)$ i is meet-distributive, then the corresponding operation $\oplus$ distributes over arbitrary joins: $a \oplus\left(\bigvee_{i \in I} b_{i}\right)=\bigvee_{i \in I}\left(a \oplus b_{i}\right) \forall a \in L, \forall\left\{b_{i}: i \in I\right\} \subseteq L$.

Example 2.4 Important examples of De Morgan quantales are the following three. Let $L=[0,1]$ be the unit interval viewed as the lattice with the standard "less or equal" relation $\leq$ :

$*_{M}$ defined by $a *_{M} b=a \wedge b$ is the minimum $t$-norm; $*_{L} \quad$ defined by $a *_{L} b=\min (a+b-1,0)$ is the Łukasiewicz $t$-norm;

$*_{P}$ defined by $a *_{P} b=a \cdot b$, is the product $t$-norm.

Further let ${ }^{c}: L \rightarrow L$ be the standard negation, that is $a^{c}=1-a$ for every $a \in L$. Then $\left(L, \leq, \wedge, \vee, *_{\wedge}\right)$, $\left(L, \leq, \wedge, \vee, *_{L}\right),\left(L, \leq, \wedge, \vee, *_{P}\right)$, are meet-distributive De Morgan quantales.

2.3. Girard quantales In order to deal with duality between operators of erosion and dilation, it is important that negation ${ }^{c}$ in a quantale is well-coordinated 
with the original quantale structure $(L, \leq, \wedge, \vee, *)$. Explicitly, this means that the negation should be defined according to the laws of fuzzy logic, that is $a^{c}=a \mapsto 0$. Therefore, to satisfy the properties of the negation, considering the problem of duality we have to request that $(a \mapsto 0) \mapsto 0=a$ for every $a \in L$. Quantales $(L, \leq, \wedge, \vee, *)$ satisfying this property are called Girard quantales, cf [12], [11], [6]. An important example of Girard quantales are MV-algebras. Obviously a Girard quantale is a special kind of a De Morgan quantale. In particular, the De Morgan quantale $\left(L, \leq, \wedge, \vee, *_{L}\right)$ defined above is a Girard quantale.

We will need also the following Lemma:

Lemma 2.5 In a Girard quantale $(L, \leq, \wedge, \vee, *) \quad a \mapsto$ $b=(a *(b \mapsto 0)) \mapsto 0$. for all $a, b \in L$.

Proof Given $a, b \in L$, we get $a *(b \mapsto 0) \mapsto 0=\bigvee\{\lambda \mid$ $\lambda * a *(b \mapsto 0) \leq 0\}=\bigvee\{\lambda \mid \lambda * a \leq(b \mapsto 0) \mapsto 0\}=$ $\bigvee\{\lambda \mid \lambda * a \leq b\}=a \mapsto b$.

Corollary 2.6 In a Girard quantale $a *(b \mapsto 0)=$ $(a \mapsto b) \mapsto 0$ for any $a, b \in L$.

2.4 $L$-fuzzy relations Let $L$ be a quantale, $X$ be a set and $R: X \times X \rightarrow L$ be an $L$-fuzzy relation (see e.g. [17],[16]). An $L$ - fuzzy relation $R$ on a set $X$ is called reflexive if $R(x, x)=1_{L}$ for every $x \in X$; An $L$-fuzzy relation $R$ on a set $X$ is called symmetric, if $R(x, y)=$ $R(y, x)$ for all $x, y \in X$; An $L$-fuzzy relation $R$ on a set $X$ is called transitive, if $R(x, y) * R(y, z) \leq R(x, z)$ for all $x, y, z \in X$. In what follows all $L$-fuzzy relations are assumed to be reflexive.

\section{Structured $L$-fuzzy relational erosion and dilation}

\subsection{Definitions and basic properties}

Let $X$ be a set and $R: X \times X \rightarrow L$ be an $L$-fuzzy relation. Modifying the definition of $L$-fuzzy relational erosion given in [9], see also [14] to the case when the fuzzy erosion of a fuzzy set $A \in L^{X}$ is structured by a fuzzy set $B \in L^{Y}$, we come to the following definition:

Definition 3.1 Let $A, B \in L^{X}$. The erosion of A structured by $B$ in the space $(X, R)$ is the L-fuzzy set $\mathscr{E}_{R}(A, B) \in L^{X}$ defined for every $y \in X$ by

$\mathscr{E}_{R}(A, B)(y)=\bigwedge_{x \in X}(R(x, y) \vee B(x) \mapsto A(x))$.

Considering erosion for all $A \in L^{X}$ when $B \in L^{X}$ is fixed, we get operator of erosion $\mathscr{E}_{R}(\cdot, B): L^{X} \rightarrow L^{X}$

In the next proposition we collect some properties of erosion operators.
Proposition 3.2 Let $R: X \times Y \rightarrow L$ be an L-fuzzy relation. Then

(1) $\mathscr{E}_{R}(A, B) \leq$ A for every $A \in L^{X}$;

(2) $\mathscr{E}_{R}\left(a_{X}, B\right)=a_{X}$ where $a_{X}$ is the constant fuzzy set with value $a \in L$;

(3) if $A_{1} \leq A_{2} \in L^{X}$ then $\mathscr{E}_{R}\left(A_{1}, B\right) \leq \mathscr{E}_{R}\left(A_{2}, B\right)$;

(4) if $B_{1} \leq B_{2} \in L^{X}$ then $\mathscr{E}_{R}\left(A, B_{1}\right) \geq \mathscr{E}_{R}\left(A, B_{2}\right)$ for every $A \in L^{X}$;

(5) Given a family $\left\{A_{i} \mid i \in I\right\} \subseteq L^{X}$ and $B \in L^{X}$, it holds $\mathscr{E}_{R}\left(\bigwedge_{i \in I} A_{i}, B\right)=\bigwedge_{i \in I} \mathscr{E}_{R}\left(A_{i}, B\right)$.

Proof $(1) \mathscr{E}_{R}(A, B)(y)=\bigwedge_{x}(R(x, y) \vee B(x) \mapsto A(x)) \leq$ $R(y, y) \vee B(y) \mapsto A(y)=A(y)$.

(2) $\mathscr{E}_{R}\left(a_{X}, B\right)(y)=\bigwedge_{x \in X}\left(R(x, y) \vee B(x) \mapsto a_{X}\right)=$ $\left(\bigvee_{x}(R(x, y) \vee B(x)) \mapsto a=1 \mapsto a=a_{X}\right.$.

The proof of the third and fourth statements follows directly from the definitions and the properties of the residuum.

For the proof of the fifth statement let a family $\left\{A_{i} \mid i \in I\right\} \subseteq L^{X}$ and $y \in X$ be given. Applying 2.1 we have $\varepsilon_{R}\left(\bigwedge_{i \in I} A_{i}, B\right)(y)=$ $\bigwedge_{x \in X}\left(\bigwedge_{i \in I}\left(R(x, y) \vee B(x) \mapsto A_{i}(x)\right)\right)$ $\bigwedge_{i \in I}\left(\bigwedge_{x \in X}\left(R(x, y) \vee B(x) \mapsto A_{i}(x)\right)\right)$ $\bigwedge_{i \in I} \mathscr{E}_{R}\left(A_{i}, B\right)(y)$

Proposition 3.3 If L-fuzzy relation $R$ is transitive, and $B$ is a constant fuzzy set then $\mathscr{E}_{R}\left(\mathscr{E}_{R}(A, B) B\right)=$ $\mathscr{E}_{R}(A, B)$ and hence operator $\mathscr{E}_{R}(\cdot, B)$ is idempotent.

Proof Let $z \in X$ then $\mathscr{E}_{R}\left(\mathscr{E}_{R}(A, B), B\right)(z)=$ $\bigwedge_{y \in X}\left(R(y, z) \vee B(y) \mapsto \mathscr{E}_{R}(A, B)\right)=$

$\bigwedge_{y \in X}\left(R(y, z) \vee B(y) \mapsto\left(\bigwedge_{x \in X}(R(x, y) \vee B(x) \mapsto A)\right)\right)=$

$\bigwedge_{x, y \in X}(R(y, z) \vee B(y) \mapsto(R(x, y) \vee B(x) \mapsto A))=$

$\bigwedge_{x, y \in X}(R(y, z) \vee B(y) *(R(x, y) \vee B(x) \mapsto A))=$

$\bigwedge_{x, y \in X}((R(y, z) *(R(x, y)) \vee(B(y) \vee B(x)) \mapsto A)) \geq$

$\left.\left.\bigwedge_{x, \in X}(R(x, z) \vee B(x)) \mapsto A\right)\right)=\mathscr{E}_{R}(A, B)(z)$.

and hence $\mathscr{E}_{R}\left(\mathscr{E}_{R}(A, B) B\right)(z) \geq \mathscr{E}_{R}(A, B)(z)$. Since the opposite inequality follows from Proposition 3.1 we get the required equality $\mathscr{E}_{R}\left(\mathscr{E}_{R}(A, B) B\right)=\mathscr{E}_{R}(A, B)$

Turning to the structured version of dilation, let $X$ be a set and $R: X \times X \rightarrow L$ an $L$-fuzzy relation.

Definition 3.4 Given $A \in L^{X}$, its $L$-fuzzy dilation structured by $B \in L^{X}$ is an L-fuzzy set $\mathscr{D}_{R}(A, B) \in$ $L^{X}$ for every $y \in X$ defined by $\mathscr{D}_{R}(A, B)(y)=$ $\bigvee_{x \in X}(R(y, x) \vee B(x)) * A(x)$.

Considering dilation for all $A \in L^{X}$ we get operator of dilation $\mathscr{D}_{R}(\cdot, B): L^{X} \rightarrow L^{X}$.

In the next proposition we collect basic properties of dilation operator $\mathscr{D}_{R}(\cdot, B)$.

Proposition 3.5 Let $R: X \times Y \rightarrow L$ be an $L$-fuzzy relation. Then

(1) $\mathscr{D}_{R}(A, B) \geq A$ for every $A \in L^{X}$; 
(2) $\mathscr{D}_{R}\left(a_{X}, B\right)=a_{X}$;

(3) $A_{1} \leq A_{2} \in L^{X} \Longrightarrow \mathscr{D}_{R}\left(A_{1}, B\right) \leq \mathscr{D}_{R}\left(A_{2}, B\right)$;

(4) if $B_{1} \leq B_{2} \in L^{X}$, then for every $A \in L^{X}$ $\mathscr{D}_{R}\left(A, B_{1}\right) \leq \mathscr{D}_{R}\left(A, B_{2}\right)$;

(5) Given a family of L-fuzzy sets $\left\{A_{i} \mid i \in I\right\} \subseteq L^{X}$, it holds $\mathscr{D}_{R}\left(\bigvee_{i \in I} A, B\right)=\bigvee_{i \in I} \mathscr{D}_{R}\left(A_{i}, B\right)$.

Proof The proof of the first four properties follows directly from the definitions. The proof of the $5^{\text {th }}$ property follows from the next equalities:

$\left.\mathscr{D}_{R}\left(\bigvee_{i \in I} A_{i}, B\right)=\bigvee_{x \in X}(R(y, x) \vee B(x)) *\left(\bigvee_{i \in I} A_{i}\right)\right)=$ $\left.\bigvee_{x \in X}\left(\bigvee_{i \in I}(R(y, x) \vee B(x)) * A_{i}\right)\right)$

$\left.\bigvee_{i \in I}\left(\bigvee_{x \in X}(R(y, x) \vee B(x)) * A_{i}\right)\right)=\bigvee_{i \in I} \mathscr{D}\left(A_{i}, B\right)$

From propositions 3.2 and 3.5 follows corollary:

Corollary 3.6 $\mathscr{E}_{R}(A, B) \leq A \leq \mathscr{D}_{R}(A, B)$.

Theorem 3.7 If $R$ is transitive and $B$ is a constant fuzzy set, then $\mathscr{D}_{R}\left(\mathscr{D}_{R}(A, B), B\right)=\mathscr{D}_{R}(A, B)$ for every $A \in L^{X}$

Proof Let $z \in X$. Then $\mathscr{D}_{R}\left(\mathscr{D}_{R}(A, B), B\right)(z)=$ $\bigvee_{y \in X}(R(z, y) \quad \vee \quad B(y)) \quad * \quad\left(\mathscr{D}_{R}(A, B)\right)(y)=$ $\bigvee_{y \in X}\left((R(z, y) \vee B(y)) *\left(\bigvee_{x \in X}(R(y, x) \vee B(x)) * A(x)\right)\right)=$ $\left.\bigvee_{x, y \in X}(R(z, y) \vee B(y)) *(R(y, x) \vee B(x)) * A(x)\right) \leq$ $\bigvee_{x, \in X}(R(z, x) \vee B(x)) * A(x)$, and hence $\mathscr{D}_{R}\left(\mathscr{D}_{R}(A, B), B\right)(z) \leq \mathscr{D}_{R}(A, B)(z)$ for every $z \in X$. Since the opposite inequality follows from Proposition 3.5, we get the equality $\mathscr{D}_{R}\left(\mathscr{D}_{R}(A, B), B\right)=\mathscr{D}_{R}(A, B)$.

\subsection{Interrelations between structured fuzzy relational erosion and dilation}

One of the most important attributes of mathematical morphology is the interrelations between erosion and dilation which manifest in two ways: as the adjunction between erosion and dilation and as the duality between erosion and dilation.

Theorem 3.8 Let $\left(L, \leq, \vee, \wedge, *{ }^{c}\right)$ be a Girard quantale. Then for every $B \in L^{X}$ operators $\mathscr{E}_{R}(\cdot, B)$ and $\mathscr{D}_{R}(\cdot, B)$ make a dual pair:

$\mathscr{E}_{R}^{c}(A, B)=\mathscr{D}_{R}\left(A^{c}, B\right), \mathscr{D}_{R}^{c}(A, B)=\mathscr{E}_{R}\left(A^{c}, B\right) \quad \forall A \in L^{X}$.

Proof We prove the theorem by a series of equivalent transitions which are justified by the definition of Girard quantale and statement 2.6 :

$\mathscr{E}_{R}^{c}(A, B)(y)=\left(\bigwedge_{x \in X}(R(x, y) \vee B(x) \mapsto A(x))\right) \mapsto 0=$ $\bigvee_{x \in X}((R(x, y) \vee B(x) \mapsto A(x)) \mapsto 0)=$

$\left.\bigvee_{x \in X}(R(x, y) \vee B(x)) *(A(x) \mapsto 0)\right)=\mathscr{D}_{R}\left(A^{c}, B\right)(y)$.

The second equality can be proved in a similar way or obtained from the first one by applying the duality law.
Theorem 3.9 Let $(L, \leq, \wedge, \vee, *)$ be a quantale: Then $\left(\mathscr{E}_{R}(\cdot, B), \mathscr{D}_{R}(\cdot, B)\right)$ is an adjunctive pair.

Proof Since both operators $\mathscr{E}_{R}(\cdot, B)$ and $\mathscr{D}_{R}(\cdot, B)$ are defined and take values in the same lattice $L^{X}$, the adjunction just means that for any $A, C \in L^{X}$ :

$A \leq \mathscr{E}_{R}(C, B) \Longleftrightarrow \mathscr{D}_{R}(A, B) \leq C$.

We prove this by the following series of transitions; the transition from the third to the fourth line is justified by replacing $x$ with $y$ and vice versa. Let $y \in X$. Then

$\mathscr{D}_{R}(A, B)(y) \leq C(y) \forall y \in X \Longleftrightarrow$

$\left(\bigvee_{x \in X}(R(y, x) \vee B(x)) * A(x)\right) \leq C(y) \forall y \in X \Longleftrightarrow$

$((R(y, x) \vee B(x)) * A(x)) \leq C(y) \forall x, y \in X \Longleftrightarrow$

$(R(x, y) \vee B(y)) * A(y) \leq C(x) \forall x, y \in X \Longleftrightarrow$

$A(y) \leq(R(x, y) \vee B(y)) \mapsto C(x) \forall x, y \in X \Longleftrightarrow$

$A(x) \leq \bigwedge_{y \in X}((R(y, x) \vee B(x)) \mapsto C(x)) \forall x, \in X \Longleftrightarrow$

$A(x) \leq \mathscr{E}_{R}(C, B)(x) \forall x \in X \Longleftrightarrow A \leq \mathscr{E}_{R}(C, B)$.

\section{Aggregation of structured fuzzy relational erosion and fuzzy relational dilation}

In order to develop an approach for the aggregation problem in fuzzy morphology we have first to choose an aggregation operator $\diamond$ that in the context of this work will be a mapping $\diamond: L \times L \rightarrow L$ satisfying certain conditions. Different kinds of aggregation operators were defined, studied and applied by many authors, see, e.g. [2]. We start with aggregation of erosion and dilation operators on the base of operator $\wedge$ and its dual $\vee$ postponing consideration of other possible operators for aggregation to Subsection 4.3

\section{1 $(\wedge, \vee)$-aggregation}

Our first choice is to aggregate on the basis of the dual min-max pair, that we call $(\wedge, \vee)$-aggregation.

Let $(L, \leq, \wedge, \vee, *)$ be, as usually, a quantale, $X$ be a set and $R: X \times X \rightarrow L$ an $L$-fuzzy relation. Further, let $\left\{B_{k}: k=1, \ldots, n\right\} \subseteq L^{X}$ be a family of $L$-fuzzy sets and $\mathscr{E}_{R}\left(\cdot, B_{k}\right): L^{X} \rightarrow L^{\bar{X}}$, be the corresponding erosion operators. Since one can anticipate that the simultaneous action of several erosion operators $\mathscr{E}_{R}\left(\cdot, B_{k}\right): L^{X} \rightarrow L^{X}$ should increase the result of the erosion, that is diminish the image of the original fuzzy set $A$, we suggest to aggregate the erosion operators $\mathscr{E}_{R}\left(\cdot, B_{k}\right)$ by taking their minimum, that is to define the resulting erosion operator by $\mathscr{E}_{R}(\cdot, B)^{1}=\bigwedge_{i=k}^{n} \mathscr{E}_{R}\left(\cdot, B_{k}\right)$. As the next theorem shows, $\mathscr{E}_{R}(\cdot, B)$ is just the erosion realized by relation $R$ and the structuring element $B=\bigvee_{k=1}^{n} B_{i}$.

Theorem 4.1 $\mathscr{E}_{R}\left(\cdot, \bigvee_{k=1}^{n}\left(\cdot, B_{k}\right)\right)=\bigwedge_{k=1}^{n} \mathscr{E}_{R}\left(\cdot, B_{k}\right)$

\footnotetext{
${ }^{1}$ Theorem 4.1 will justify such notation
} 
Proof It is sufficient to prove the statement in case of two aggregated erosion operators $\mathscr{E}_{R}\left(\cdot, B_{1}\right)$ and $\mathscr{E}_{R}\left(\cdot, B_{2}\right)$; the general case will follow by induction. Let $A \in L^{X}$ and $y \in X$ be given. Then applying wellknown properties of the residuum $\mapsto$, (see e.g. [5], cf also [8], etc.) we have:

$$
\begin{aligned}
& \mathscr{E}_{R}\left(A, B_{1}\right) \wedge \mathscr{E}_{R}\left(A, B_{2}\right)= \\
& \left(\bigwedge_{x \in X}\left(R(x, y) \vee B_{1}(x) \mapsto A(x)\right)\right) \wedge \\
& \left(\bigwedge_{x \in X}\left(R(x, y) \vee B_{2}(x) \mapsto A(x)\right)\right)= \\
& \bigwedge_{x \in X}\left(\left(R(x, y) \vee B_{1}(x) \mapsto A(x)\right) \wedge\right. \\
& \left.\left(R(x, y) \vee B_{2}(x) \mapsto A(x)\right)\right)= \\
& \bigwedge_{x \in X}\left(\left(R(x, y) \vee B_{1}(x)\right) \vee\left(R(x, y) \vee B_{2}(x)\right) \mapsto A(x)\right)= \\
& \bigwedge_{x \in X}\left(\left(R(x, y) \vee B_{1}(x) \vee B_{2}(x)\right) \mapsto A(x)\right)= \\
& \bigwedge_{x \in X}\left(\left(R(x, y) \vee\left(B_{1} \vee B_{2}\right)(x)\right) \mapsto A(x)\right)= \\
& \mathscr{E}_{R}\left(A, B_{1} \vee B_{2}\right) \quad \square
\end{aligned}
$$

Corollary 4.2 Operator obtained by $\wedge$-aggregation of a family of fuzzy relational erosion operators structured by $B_{k}, k=1, \ldots, n$ is a fuzzy relational erosion operator structured by $B=\bigvee_{k=1}^{n} B_{k}$, that is $\mathscr{E}_{R}(\cdot, B)$.

In order to ensure one of the principal requests of the mathematical morphology, namely, to provide duality between aggregated erosion and dilation operators, we have to define aggregation of dilation operators on the base of the $V$ operation. This seems also to be natural, since simultaneous action of several dilations is expected to strengthen their result, that is to enlarge the original image in the result of aggregating dilations.

Let $B_{k}: X \times X \rightarrow L, k=1, \ldots n$ be a family of $L$ fuzzy sets, $R: X \times X \rightarrow L$ be an $L$-fuzzy relation and $\mathscr{D}_{R}\left(\cdot, B_{k}\right): L^{X} \rightarrow L$, be the corresponding dilation operators. We define the dilation $\mathscr{D}_{R}$ obtained by aggregation of $\mathscr{D}_{R}\left(\cdot, B_{k}\right): L^{X} \rightarrow L$ as its supremum, that is $\mathscr{D}_{R}(\cdot, B)^{2}=\bigvee_{k=1}^{n} \mathscr{D}_{R}\left(\cdot, B_{k}\right)$.

Theorem 4.3 $\mathscr{D}_{R}\left(\cdot, \bigvee_{k=1}^{n} B_{k}\right)=\bigvee_{k=1}^{n} \mathscr{D}_{R}\left(\cdot, B_{k}\right)$.

Proof Again, we restrict to the case when two dilation operators $\mathscr{D}_{R}\left(\cdot, B_{1}\right)$ and $\mathscr{D}_{R}\left(\cdot, B_{2}\right)$ are aggregated. Given $A \in L^{X}$ and $y \in X$ we have:

$$
\begin{aligned}
& \left(\mathscr{D}_{R}\left(A, B_{1}\right) \vee \mathscr{D}_{R}\left(A, B_{2}\right)\right)(y)= \\
& \mathscr{D}_{R}\left(A, B_{1}\right)(y) \vee \mathscr{D}_{R}\left(A, B_{2}\right)(y)= \\
& \left.\left(\bigvee_{x \in X}\left(R(x, y) \vee B_{1}(x)\right) * A(x)\right)\right) \vee \\
& \left(\bigvee_{x \in X}\left(R(x, y) \vee B_{2}(x)\right) * A(x)\right)= \\
& \bigvee_{x \in X}\left(\left(\left(R(x, y) \vee B_{1}(x)\right) * A(x)\right) \vee\right. \\
& \left.\left(\left(R(x, y) \vee B_{2}(x)\right) * A(x)\right)\right)= \\
& \bigvee_{x \in X}\left(\left(R(x, y) \vee B_{1}(x) \vee B_{2}(x)\right) * A(x)\right)= \\
& \mathscr{D}_{R}\left(A, B_{1} \vee B_{2}\right)(x), \\
& \text { and hence } \mathscr{D}_{R}\left(A, B_{1}\right) \vee \mathscr{D}_{R}\left(A, B_{2}\right)= \\
& \mathscr{D}_{R}\left(A, B_{1} \vee B_{2}\right) .
\end{aligned}
$$

\footnotetext{
${ }^{2}$ Theorem 4.3 justifies such notation
}

Since $(\wedge, \vee)$-aggregated fuzzy relational erosion and dilation operators remain fuzzy relational erosion and dilation, they satisfy all properties established for such operators in Section 2 and Section 3. Especially we highlight the following:

Theorem 4.4 [Duality] Let $L$ be a Girard quantale, $R: X \times X \rightarrow L$, be an $L$-fuzzy relation and $\left\{B_{k} \mid k=\right.$ $1, \ldots n\} \subseteq L^{X}$ be a family of $L$-fuzzy sets. Then for every $A \in L^{X}$

$$
\bigwedge_{k=1}^{n} \mathscr{E}_{R}\left(A^{c}, B_{k}\right)=\left(\bigvee_{k=1}^{n} \mathscr{D}_{R}\right)^{c}\left(A, B_{k}\right)
$$

Theorem 4.5 [Adjunction] Let $L$ be a quantale, $R$ : $X \times X \rightarrow L$, be an $L$-fuzzy relation and $\left\{B_{k} \mid k=\right.$ $1, \ldots n\} \subseteq L^{X}$ be a family of $L$-fuzzy sets. Then $\left(\bigwedge_{k=1}^{n} \mathscr{E}_{R}\left(\cdot, B_{k}\right), \bigvee_{k=1}^{n} \mathscr{D}_{R}\left(\cdot, B_{k}\right)\right)$ is an adjunctive pair, that is, for any $A, B, C \in L^{X}$

$$
\left.\bigwedge_{k=1}^{n} \mathscr{E}_{R}\left(A, B_{k}\right) \leq C \Longleftrightarrow A \leq \bigvee_{k=1}^{n} \mathscr{D}_{R}\left(C, B_{k}\right)\right)
$$

\section{$4.2(\vee, \wedge)$-aggregation.}

One can ask what will be obtained if we interchange the roles of $\wedge$ and $\vee$ in defining aggregation of erosions and dilations. Namely, aggregate erosions by $\vee$ and aggregate dilations by $\wedge$. However, we do not see any practical motivation for this: the joint use of erosions is expected to strengthen both the erosion and dilation, not weaken them as it will happen in the discussed case. And second, in this case we will have only the trivial (as obvious corollaries of propositions 3.2 and 3.5 ) results, namely:

Proposition 4.6 Let $R: X \times X \rightarrow L$ and let $\left\{B_{1}, \ldots, B_{n}\right\}$ be a family of L-fuzzy sets. Then $\mathscr{E}_{R}\left(\cdot, \bigwedge_{k=1}^{n} B_{k}\right) \geq \bigvee_{k=1}^{n} \mathscr{E}_{R}\left(\cdot, B_{k}\right)$; $\mathscr{D}_{R}\left(\cdot, \bigvee_{k=1}^{n} B_{k}\right) \geq \bigwedge_{k=1}^{n} \mathscr{D}_{R}\left(\cdot, B_{k}\right)$.

Nevertheless also this aggregation process preserves the duality and adjunctness of the obtained operators.

Proposition 4.7 Let $(L, \leq, \wedge, \vee, *)$ be a Girard quantale.Then for all $A, B_{k}, \in L^{X}$

$\bigvee_{k=1}^{n} \mathscr{E}_{R}\left(A^{c}, B_{i}\right)=\left(\bigwedge_{k=1}^{n} \mathscr{D}_{R}\right)^{c}\left(A, B_{k}\right)$,

$\left(\bigvee_{k=1}^{n} \mathscr{E}_{R}\right)^{c}\left(A, B_{k}\right)=\bigwedge_{i=1}^{n} \mathscr{D}_{R_{i}}\left(A^{c}, B_{k}\right)$.

The proof follows directly from the definitions.

Proposition 4.8 Let $(L, \leq, \wedge, \vee, *)$ be a linearly ordered quantale. Then for all $A, B_{i}, C \in L^{X}$ $\bigvee_{i=1}^{n} \mathscr{E}_{R}\left(A, B_{i}\right) \leq C \Longleftrightarrow A \leq \bigwedge_{i=1}^{n} \mathscr{D}_{R}\left(C, B_{i}\right)$.

Proof We prove the statement in case of two operators. Let $\mathscr{D}\left(A, B_{1}\right) \wedge \mathscr{D}\left(A, B_{2}\right) \leq C$ and $y \in Y$. Then $\mathscr{D}\left(A, B_{1}\right)(y) \wedge \mathscr{D}\left(A, B_{2}\right)(y) \leq C(y)$. Without loss of 
generality assume that $\left.\mathscr{D}\left(A, B_{1}\right)(y)\right) \leq C(y)$. Then referring to the proof of theorem 3.9, $A(x) \leq \mathscr{E}\left(A, B_{1}\right)(x)$ and hence $A(x) \leq \mathscr{E}\left(A, B_{1}\right)(x) \vee \mathscr{E}\left(A, B_{2}\right)(x)$ for every $x \in X$, that is, $A \geq \mathscr{E}\left(A, B_{1}\right) \vee \mathscr{E}\left(A, B_{2}\right)$.

\subsection{Aggregation of fuzzy morphological operators: the general approach}

As in the previous subsections, we consider structured $L$-fuzzy erosion and dilation operators $\mathscr{E}_{R}\left(\cdot, B_{k}\right): L^{X} \rightarrow$ $L^{X}$ and $\mathscr{D}_{R}\left(\cdot, B_{k}\right): L^{X} \rightarrow L^{X}$ where $\left(L, \leq, \wedge, \vee, *{ }^{c}\right)$ is a De Morgan quantale, $R: X \times X \rightarrow L$ is a reflexive $L$ fuzzy relation and $B_{k} \in L^{X}$. In the previous subsections we considered aggregation of such operators by means of the $\wedge$ and $\vee$ operations on $\left(L, \leq, \wedge, \vee, *{ }^{c}\right)$. In this and the next subsection we analyse the perspectives to aggregate erosion and dilation operators on the base of alternative pairs of operators $(\diamond, \odot)$, where $\diamond$ is a binary operation on $L$ and $\odot$ is its dual, that is, $a \odot$ $b=\left(a^{c} \diamond b^{c}\right)^{c}$. We assume that $\diamond$ satisfies the following properties:

$\left(\diamond_{1}\right) 1_{L} \diamond a=a \forall a \in L$

$\left(\diamond_{2}\right) a \leq b \Longrightarrow a \diamond c \leq a \diamond c \leq b \diamond c \forall a, b, c \in L$

$\left(\diamond_{3}\right) a \diamond b=b \diamond a \forall a, b \in L$

$\left(\diamond_{4}\right)(a \diamond b) \diamond c=a \diamond(b \diamond c) \forall a, b, c \in L$.

From the properties of $\diamond$ we obtain the corresponding properties for its dual operator $\odot$ :

$\left(\odot_{1}\right) 0_{L} \odot a=a \forall a \in L$;

$\left(\odot_{2}\right) a \leq b \Longrightarrow a \odot c \leq b \odot c \forall a, b, c \in L$

$\left(\odot_{3}\right) a \odot b=b \odot a \forall a, b \in L$;

$\left(\odot_{4}\right)(a \odot b) \odot c=a \odot(b \odot c) \forall a, b, c \in L$

We define aggregation of erosion and dilation operators by means of the pair $(\diamond, \odot)$ of operators as follows. Let $R: X \times X \rightarrow L$ be an $L$-fuzzy relation and $\left\{B_{k} \mid k=1, \ldots, n\right\} \subseteq L^{X}$ be a family of $L$-fuzzy sets.

Definition 4.9 Let $\mathscr{E}_{R}\left(\cdot, B_{k}\right): L^{X} \rightarrow L^{X}$, be fuzzy relational erosion operators structured by L-fuzzy sets $B_{k}$. We define aggregation of these operators by $\diamond_{k=1}^{n} \mathscr{E}_{R}\left(\cdot, B_{k}\right):\left(L^{X}\right)^{n} \rightarrow L^{X}$, or explicitly,

$\mathscr{E}_{R}\left(A, B_{1}\right)(y) \diamond, \ldots, \diamond \mathscr{E}_{R}\left(A, B_{n}\right)(y) \forall A \in L^{X}, y \in X$.

From Proposition 3.2 we easily can get the following properties of the aggregated erosion operator:

\section{Proposition 4.10}

(1) $\diamond_{k=1}^{n} \mathscr{E}_{R}\left(A, B_{k}\right) \leq A$ for every $A \in L^{X}$;

(2) $\diamond_{k=1}^{n} \mathscr{E}_{R}\left(a_{X}, B_{i}\right)=a_{X}$;

(3) If $A_{1} \leq A_{2} \in L^{X}$ then $\diamond_{k=1}^{n} \mathscr{E}_{R}\left(A_{1}, B_{k}\right) \leq$ $\diamond_{k=1}^{n} \mathscr{E}_{R}\left(A_{2}, B_{k}\right)$;

(4) If $B_{k} \leq B_{k}^{\prime} \in L^{X}$ for every $k=1, \ldots, n$, then for every $A \in L^{X} \diamond_{k=1}^{n} \mathscr{E}_{R}\left(A, B_{k}\right) \geq \diamond_{k=1}^{n} \mathscr{E}_{R}\left(A, B_{k}^{\prime}\right)$.
Definition 4.11 Let $\mathscr{D}_{R}\left(\cdot, B_{k}\right): L^{X} \rightarrow L^{X}$, be fuzzy relational dilation operators structured by L-fuzzy sets $B_{k}$. We define aggregation of these operators by $\bigodot_{k=1}^{n} \mathscr{D}_{R}\left(\cdot, B_{k}\right):\left(L^{X}\right)^{n} \rightarrow L^{X}$, or explicitly

$\mathscr{D}_{R}\left(A, B_{1}\right)(y) \odot, \ldots, \odot \mathscr{D}_{R}\left(A, B_{n}\right)(y) \forall A \in L^{X}, y \in X$.

From Proposition 3.5 we easily get the following properties of the aggregated dilation operator:

\section{Proposition 4.12}

(1) $\bigodot_{k=1}^{n} \mathscr{D}_{R}\left(A, B_{k}\right) \geq A$ for every $A \in L^{X}$;

(2) $\bigodot_{k=1}^{n} \mathscr{D}_{R}\left(a_{X}, B_{k}\right)=a_{X}$;

(3) If $A_{1} \leq A_{2} \in L^{X}$ then $\bigodot_{k=1}^{n} \mathscr{D}_{R}\left(A, B_{k}\right) \leq$ $\bigodot_{k=1}^{n} \mathscr{D}_{R}\left(A, B_{k}\right)$.

Thanks to the assumed duality of the pair $(\diamond, \odot)$ we have the following important duality between the aggregated operators:

\section{Theorem 4.13}

$\diamond_{k=1}^{n} \mathscr{E}_{R}\left(A^{c}, B_{k}\right)=\bigodot_{k=1}^{n} \mathscr{D}_{R}^{c}\left(A, B_{k}\right)$ for every $A \in L^{X}$; $\bigodot_{k=1}^{n} \mathscr{D}_{R}\left(A^{c}, B_{k}\right)=\diamond_{k=1}^{n} \mathscr{E}_{R} c\left(A, B_{k}\right)$ for every $A \in L^{X}$.

Proof We can restrict the proof for the case of aggregation of two operators. Thus to prove the first equality we have to show that the equality $\mathscr{E}_{R}\left(A^{c}, B_{1}\right)(y) \diamond$ $\mathscr{E}_{R}\left(A^{c}, B_{2}\right)(y)=\mathscr{D}_{R}^{c}\left(A, B_{1}\right)(y) \odot \mathscr{D}_{R}^{c}\left(A, B_{n}\right)(y)$ holds for every $A \in L^{X}$.

We get it as follows. Referring to the duality of the pair $(\diamond, \odot)$ and the duality between operators $\mathscr{E}\left(\cdot, B_{k}\right)$ and $\mathscr{D}\left(\cdot, B_{k}\right)$, see Theorem 3.8, for every $A \in L^{X}$ and every $y \in X$ we have

$\left(\mathscr{E}_{R}\left(A^{c}, B_{1}\right)(y) \diamond \mathscr{E}_{R}\left(A^{c}, B_{2}\right)(y)\right)^{c}=\mathscr{E}_{R}^{c}\left(A^{c}, B_{1}\right)(y) \odot$ $\left.\mathscr{E}_{R}^{c}\left(A^{c}, B_{2}\right)(y)=\mathscr{D}_{R}\left(A^{c}, B_{1}\right)(y) \odot \mathscr{D}_{R}\left(A, B_{2}\right)(y)\right)$.

Form this equality we get the requested one just by replacing $A^{c}$ with $A$.

The second equality can be proved similarly or obtained from the first one by referring to duality of $(\diamond, \odot)$.

\subsection{Additional properties of $(\diamond, \odot)$-aggregated erosion and dilation operators}

Going further with the study of operators obtained by aggregation erosions and dilations, first we have to note the following important fact. As different from the case of $(\wedge \vee)$-aggregation, generally we do not know whether the operators obtained by $(\diamond, \odot)$ are indeed respectively structured fuzzy relational erosion and dilation operators. Therefore for $(\diamond, \odot)$ aggregated operators we will use notations not specifying an $L$-fuzzy relation and a structuring element:

$$
\mathfrak{E}(A)=\diamond_{k=1}^{n} \mathscr{E}_{R}\left(A, B_{k}\right), \mathfrak{D}(A)=\bigodot_{k=1}^{n} \mathscr{D}_{R}\left(a_{X}, B_{k}\right) .
$$


Second, to state some non-trivial assertions, we additionally have to assume certain relations between operators $\diamond$ and $\odot$ used for aggregation and the original operator $*$ in the De Morgan quantale $\left(L, \leq, \wedge, \vee, *,{ }^{c}\right)$.

Patterned after definitions specified in Section 2, we call operation $\diamond$ meet continuous, if $\left(\bigwedge_{i \in I} a_{i}\right) \diamond b=$ $\bigwedge_{i \in I}\left(a_{i} \diamond b\right)$ for any $\left\{a_{i} \mid i \in I\right\} \subseteq L, b \in L$. Obviously, by duality, meet continuity of $\diamond$ is equivalent to join continuity of the operation $\odot$, that is, $\left(\bigvee_{i \in I} a_{i}\right) \odot b=$ $\bigvee_{i \in I}\left(a_{i} \odot b\right)$ for any $\left\{a_{i} \mid i \in I\right\} \subseteq L, b \in L$.

As an example of meet continuous operation one can take any continuous $t$-norm $T:[0,1] \times[0,1] \rightarrow[0,1]$ in the role of $\diamond$ and its dual $t$-conorm in the role of $\odot$.

Theorem 4.14 Let $\left(L, \leq, \wedge, \vee{ }^{c}, *\right)$ be a De Morgan quantale and $\diamond: L \times L \rightarrow L$ be a meet-continuous; let $\mathscr{E}_{R}\left(\cdot, B_{1}\right), \ldots \mathscr{E}_{R}\left(\cdot, B_{n}\right)$ be a family of structured fuzzy relational erosion operations. Let $\diamond_{k=1}^{n} \mathscr{E}_{R}\left(\cdot, B_{k}\right)$. Further let $\left\{A_{i} \mid i \in I\right\}$ be a family of $L$-fuzzy sets. Then

$$
\diamond_{k=1}^{n} \mathscr{E}_{R}\left(\bigwedge_{i \in I} A_{i}, B_{k}\right)=\bigwedge_{i \in I}\left(\diamond_{k=1}^{n} \mathscr{E}_{R}\left(A_{i}, B_{k}\right)\right),
$$

or in concise notations, $\mathfrak{E}\left(\bigwedge_{i \in I} A_{i}\right)=\bigwedge_{i \in I} \mathfrak{E}\left(A_{i}\right)$.

Proof We prove this statement in case when two erosion operators are aggregated. Without loss of generality we may assume that the family $\mathscr{A}=\left\{A_{i} \mid i \in I\right\}$ is $\leq$-directed. By saying this we mean that if $A_{i}, A_{j} \in \mathscr{A}$, then $A_{i} \wedge A_{j} \in \mathscr{A}$. Further, referring to meet-continuity of $\diamond$ we are reasoning as follows:

$\mathfrak{E}\left(\bigwedge_{i \in I} A_{i}\right)=\mathscr{E}_{R}\left(\bigwedge_{i \in I} A_{i}, B_{1}\right) \diamond \mathscr{E}_{R}\left(\bigwedge_{i \in I} A_{i}, B_{2}\right)=$ $\bigwedge_{i \in I} \mathscr{E}_{R}\left(A_{i}, B_{1}\right) \diamond \bigwedge_{j \in I} \mathscr{E}_{R}\left(A_{i}, B_{2}\right)=\bigwedge_{i, j \in I}\left(\mathscr{E}_{R}\left(A_{i}, B_{1}\right) \diamond\right.$ $\left.\mathscr{E}_{R}\left(A_{i}, B_{2}\right)\right)=\bigwedge_{i \in I}\left(\mathscr{E}_{R}\left(A_{i}, B_{1}\right) \diamond \mathscr{E}_{R}\left(A_{i}, B_{2}\right)\right)=$ $\bigwedge_{i \in I} \mathfrak{E}\left(A_{i}\right)$.

In a similar way, or applying the duality between erosion and dilation operators (in case we are working in a Girard quantale $\left.\left(L, \leq, \wedge, \vee{ }^{c}, *, \diamond\right)\right)$ we get the following.

Theorem 4.15 Let $\left(L, \leq, \wedge, \vee{ }^{c}, \diamond\right)$ be a De Morgan quantale and $\odot$ be join-continuous; let $\mathscr{D}_{R}\left(\cdot, B_{1}\right), \ldots$, $D_{R}\left(\cdot, B_{n}\right)$ be a family of structured fuzzy relational dilation operations and let $\bigodot_{k=1}^{n} \mathscr{D}_{R}\left(\cdot, B_{k}\right)$ be the aggregated operator. Further let $\left\{A_{i} \mid i \in I\right\}$ be a family of L-fuzzy sets. Then

$$
\bigodot_{k=1}^{n} \mathscr{D}_{R}\left(\bigvee_{i \in I} A_{i}, B_{k}\right)=\bigvee_{i \in I}\left(\bigodot_{k=1}^{n} \mathscr{E}_{R}\left(A_{i}, B_{k}\right)\right),
$$

or, in concise notations, $\mathfrak{D}\left(\bigvee_{i \in I} A_{i}\right)=\bigvee_{i \in I} \mathfrak{D}\left(A_{i}\right)$.

Remark 4.16 From Proposition 4.9, Theorem 4.14, and referring to known results (see e.g. Definition 14.16 and Theorem 14.17 in [1]), we conclude that $\mathfrak{E}$ indeed can be considered as an erosion operator that determines an adjunctive pair $\left(\mathfrak{E}, \mathfrak{D}_{\mathfrak{E}}\right)$ with some dilation operator $\mathfrak{D}_{\mathfrak{E}}$. Similarly, from Proposition 4.11 and Theorem 4.15 we conclude that $\mathfrak{D}$ indeed can be considered as a dilation operator that determines an adjunctive pair $\left(\mathfrak{E}_{\mathfrak{D}}, \mathfrak{D}\right)$ with some erosion operator $\mathfrak{E}_{\mathfrak{D}}$. Unfortunately, generally we cannot state that $\mathfrak{D}_{\mathfrak{E}}=\mathfrak{D}$ and $\mathfrak{E}_{\mathfrak{D}}=\mathfrak{E}$ as it was in case of $\diamond=\wedge$ and $\odot=\vee$.

Reasoning on the problem of aggregation fuzzy morphological operators, in particular regarding the previous remark, it seems natural to be curious about the connections between the result of aggregation of structured erosion and dilation operators and operations with corresponding structuring elements. Theorems 4.1 and 4.3 above give an exhaustive answer to this question in case when aggregation is realised by the pair $(\wedge, \vee)$. Unfortunately, concerning aggregation by other pairs $(\wedge, \vee)$, we have only the following partial and easily provable result:

Proposition 4.17 In case of Łukasiewicz t-norms * and $\diamond$

$\mathscr{D}_{R}\left(A, B_{1}\right) \odot \mathscr{D}_{R}\left(A, B_{2}\right) \geq \mathscr{D}\left(A,\left(B_{1} \cdot B_{2}\right)\right)$

$\mathscr{E}_{R}\left(A, B_{1}\right) \odot \mathscr{E}_{R}\left(A, B_{2}\right) \leq \mathscr{E}\left(A,\left(B_{1} \diamond B_{2}\right)\right)$.

Of course, the analogous statement holds also for aggregation of any finite family of operators.

\section{Conclusion}

In this paper, we introduced the structured versions of $L$-fuzzy relational erosion and dilation and studied their basic properties. Our principal interest here was aggregation of these operators. We realised such aggregation on the basis of a pair of dual binary operators. Investigating aggregation of erosion and dilation, we focused on preservation of the most important properties of these operators, specifically duality and adjunctness, in the process of aggregation. As the main challenges for the further development of aggregation theory in fuzzy morphology we view the following.

(1) We touched only aggregation of erosion and dilation. The work must be continued by including also aggregation of derived operators of fuzzy relational mathematical morphology, such as closing, opening and others.

(2) The problem to find the conditions under which aggregated relational erosion and dilation remain relational seem to us very challenging.

(3) Concerning the problem of preservation of adjunctness under aggregation: it would be very useful to find an effective description of operators $\mathfrak{D}_{\mathfrak{E}}$ and $\mathfrak{E}_{\mathfrak{D}}$.

(4) We are interested to find some practical applica- 
tions for aggregation of fuzzy relational erosion and dilation operators. Specifically, at present we are working on possible application of aggregation of fuzzy morphological operators in problems of pattern recognition. In this area fuzzy erosion and dilation operators are already effectively used and we guess that restructuring an image by aggregating fuzzy morphological operators could be helpful in this field.

(5) In this paper we stick to the fuzzy relational approach in mathematical morphology. Aggregation in the framework of other approaches to erosion and dilation operators, it particular, in the framework of algebraic as well as classical approaches should be studied.

\section{Acknowledgement}

The authors acknowledge of the partial financial support from the project No. Lzp-2020/2-0311 by the Latvian Council of Science. The authors are thankful to anonymous referees for reading the paper carefully and making useful comments. Taking them into account helped the authors to eliminate some defects and improve the exposition.

\section{References}

[1] I. Bloch, H. Hejmans, C. Ronse, Mathematical morphology, in: M. Pratt-Hartmann, J. V. Benthem (Eds.), Handbook in Spatial Logics, Springer, Berlin, 2007, pp. 857-944.

[2] T. Calvo, A. Kolesárová, M. Komorníková, R. Mesiar, Aggregation operators: Properties, classes and construction methods, in: T. Calvo, G. Mayor, R. Messiar (Eds.), Aggregation operators. New trends and applications 2002., Springer, Berlin, 2002, pp. 3-104.

[3] B. De Baets, E. Kerre, M. Gupta, The fundamentals of fuzzy mathematical morphology, part 1: basic concepts, International Journal of General Systems 23 (1995) 155-171.

[4] G. Gierz, K. Hofmann, K. Keimel, J. Lawson, M. Mislove, D. Scott, Continuous Lattices and Domains, Cambridge University Press, Cambridge, 2003.

[5] U. Höhle, $M$-valued sets and sheaves over integral commutative CL-monoids, in: S. Rodabaugh, U. Höhle, E. Klement (Eds.), Applications of Category Theory to Fuzzy Subsets, Kluwer Academic Publisher, Dodrecht, Boston, 1992, pp. 33-72.

[6] S. Jenej, Structure of Girard monoid on $[0,1]$, in: S. Rodabaugh, E. Klement (Eds.), Topological and Algebraic Structures in Fuzzy Sets, Kluwer Academic Publishers, Dodrecht, Boston, 2003, pp. 277-308.

[7] E. Kerre, M. Nachtegael, Classic and fuzzy approaches to mathematical morphology, in: E. Kerre (Ed.), Fuzzy Technique in Image Processing, Springer Verlag, Berlin, 2000, pp. 3-57.

[8] E. P. Klement, R. Mesiar, E. Pap, Triangular Norms, Vol. 8 of Trends in Logic, Kluwer Academic Publishers, Dordrecht, 2000.

[9] N. Madrid, M. Ojeda-Aciego, J. Medina, I. Perfilieva, $L$-fuzzy relational mathematical morphology based on adjoint triples, Information Sciences 474 (2019) 75-89.

[10] G. Matherron, Random sets and Integral Geometry, Willy, New York, 1974.

[11] K. Rosenthal, A note on Girard quantales, Cahiers de Topologie et Géométrie Différentielle Catégoriques 31 (1990) 3-11.

[12] K. Rosenthal, Quantales and Their Applications, Vol. 234 of Pitman Research Notes in Mathematics, Longman Scientific and Technical, 1990.

[13] J. Serra, Image analysis and mathematical morphology, Academic Press, Orlando, 1983.

[14] A. Šostak, I. Uljane, Some remarks on topological structure in the context of fuzzy relational mathematical morphology, in: Proc. of the 11th conference of the European society for fuzzy logic and technology (EUSFLAT19) Atlantis Series in Uncertainty Modelling, Vol. 1, Prague, Czech Republic, 2019, pp. 776-783.

[15] A. Šostak, I. Uljane, P. Eklund, Fuzzy relational mathematical morphology: erosion and dilation, in: Communications in Computer and Information Science, Vol. 1239, Springer, 2020, pp. 712725 .

[16] L. Valverde, On the structure of $F$ indistinguishability operators, Fuzzy Sets and Systems 17 (1985) 313-328.

[17] L. A. Zadeh, Similarity relations and fuzzy orderings, Information Sciences 3 (1971) 177-200. 